\title{
Modelling cooling of packaged fruit using 3D shape models
}

\author{
W. Gruyters ${ }^{(a)}$, P. Verboven ${ }^{(a)}$, E. Diels ${ }^{(a)}$, S. Rogge ${ }^{(a)}$, B. Smeets ${ }^{(a)}, H . R$ Ramon $^{(a)}$, T. \\ Defraeye $^{(b)}$, B. M. Nicolaï ${ }^{\left(a, c^{*}\right)}$ \\ ${ }^{a}$ BIOSYST-MeBioS, Katholieke Universiteit Leuven, Willem de Croylaan 42, B-3001 \\ Leuven, Belgium \\ ${ }^{\mathrm{b}}$ Empa, Swiss Federal Laboratories for Materials Science and Technology, Laboratory for \\ Biomimetic Membranes and Textiles, Lerchenfeldstrasse 5, 9014 St. Gallen, Switzerland \\ ${ }^{\mathrm{c}}$ Flanders Centre of Postharvest Technology, Willem de Croylaan 42, B-3001 Leuven, \\ Belgium
}

*Corresponding author;

email: bart.nicolai@ kuleuven.be - telephone number: +32 16322375

\section{Abstract}

This study presents a novel methodology to model the cooling processes of horticultural produce using realistic product shapes rather than commonly-used simplified 3D shapes, such as spheres. Variable 3D apple and pear models were created by means of a validated geometric model generator based on X-ray computed tomography images. The fruit were randomly stacked into a geometrical model of a corrugated fibreboard box using the Discrete Element Method. A forced-air cooling process was simulated for three such apple filling patterns using CFD and the results were compared to those obtained with fruit represented by equivalent spheres. No significant difference in average aerodynamic resistance between the real apple shape and its spherical representation was found. The main contributor to the overall pressure drop was the package design rather than product shape. However, large differences in local air velocity and convective heat transfer coefficients were found between the two representations. The degree of cooling uniformity between individual fruit was overestimated when using simplified product shapes: real apple fruit shapes cooled less uniform. This difference between real and simplified product shapes was even larger for a box filled with pear fruit that are more different from a spherical shape. These results 
demonstrate that improved computer-aided design approaches help in simulating more accurate convective cooling processes. In a next step, such simulations will be used for multiobjective optimization of packaging in terms of cold chain efficiency and cooling uniformity.

Keywords. computational fluid dynamics; Discrete Element Method; pome fruit; forced-air cooling; geometric model generator; biological variability

\section{INTRODUCTION}

Freshly harvested fruit are living entities that solely rely on their internal reserves to provide fuel for their respiration metabolism (Jackson, 2003). The rate at which these internal reserves are being depleted depends mainly on the temperatures to which fruit are exposed. In order to maintain fresh high-end quality produce during storage and, thus, a high economic product value, it is paramount to minimise the postharvest respiration. This is achieved right after harvest by rapidly cooling the produce to lower temperatures (Taiz et al., 2015). The common method to obtain a fast initial cooling is forced-air cooling (FAC). During this process, a pressure gradient across a stack of packages is created by a ventilator, by which refrigerated air is drawn through the packages (Brosnan and Sun, 2001).

Although a fast convective heat removal can be achieved with FAC, large temperature heterogeneities between different fruit in the ventilated package can develop during the cooling process (Alvarez and Flick, 1999; Berry et al., 2016; Han et al., 2015; Olatunji et al., 2017). The cooling rates and cooling uniformity are mainly influenced by the ease with which the cold air can penetrate into the stack of packaged fruit, and can reach the individual fruit. This, in turn, is influenced by the package design and the location of the vent holes, the fruit filling pattern inside the package but also the individual fruit size and shape, in which a distinct biological variability exists, even for the same cultivar (O'Sullivan et al., 2016). 
Investigating this complex cooling process and interactions between stacking and packaging design by means of experiments is quite cumbersome. The main reasons are that it is quite time-consuming to get a sufficient spatial resolution in cooling rates between different products, the repeatability of the experiments is difficult due to the biological variation in size and shape, and the reproducibility of the experimental setup (e.g. fruit filling pattern) is challenging.

To overcome these limitations, numerical simulations based on computational fluid dynamics (CFD) are a useful alternative to study convective cooling processes in the postharvest cold chain. It is even possible to model the realistic produce shape with techniques such as 3D scanning (Denys et al., 2003; Uyar and Erdoğdu, 2009; Van Eck et al., 1998), analytical equations (Clement et al., 2013), 2D imaging techniques (Goñi and Purlis, 2010) or X-ray computed tomography (Defraeye et al., 2012). In most recent CFD studies, the fruit shape and its size were, however, simplified with spherical products that were matched to approximate the total fruit volume inside a package (Defraeye et al., 2013a; Dehghannya et al., 2012; Delele et al., 2013; Tutar et al., 2009). Although it is feasible to extract the realistic shape of the produce, the inherent intricacies of the geometric variation in produce shapes make that using spherical geometries is more straightforward to use in a CFD model,. To improve the FAC operation of strawberries, Ferrua and Singh (Ferrua and Singh, 2011, 2009) clearly showed the added value of using realistic 3D shape models in their CFD models. However, their methodology to develop a 3D geometry of a single strawberry was based on digital images of fruit slices and involved multiple manual actions. Furthermore, the final filling pattern was developed with identical strawberries in a clamshell and was based on a manual packing procedure used in the industry. Therefore, additional manipulations were needed, which made the procedure a time-consuming endeavour. Recently, Rogge et al. (Rogge et al., 2015, 2014) developed an algorithm that generates 3D 
models of fruit in a very fast and efficient way, taking also the biological variability of shape and size into account. This algorithm has already been successfully used by Ho et al. (Ho et al., 2016) in their numerical study of gas exchange in pears during controlled atmosphere storage. They found that the internal gas concentrations were, amongst other factors, highly affected by the 3D morphology of the fruit. These studies indicate the need to take a realistic shape of the fruit into account, preferably with biological variability, in numerical simulations to gain a more accurate insight into a wide range of food processes.

The goal of this study was to develop a more accurate CFD model for studying the cooling process of freshly harvested produce stacked in a package. To this end, realistic 3D shape models of apples (cv. 'Jonagold') and pears (cv. 'Conference') were used in a Discrete Element Method simulation to generate a randomised filling pattern in a typical corrugated fibreboard package. The biological variability of the fruit is taken into account so each single fruit has a different size and shape. The resulting fruit filling pattern was compared with a package filled with spherical products. Simulations of the FAC process are performed, where cold air that is drawn through the package. Different airflow rates were considered and the effects on pressure, air velocity distribution and convective heat transfer coefficients were analysed.

\section{MATERIALS AND METHODS}

\subsection{Box design}

Compared to other telescopic corrugated fibreboard box designs, the beneficial airflow characteristics of the 'Supervent' box design have recently been demonstrated (Berry et al., 2016; Defraeye et al., 2014, 2013a). In the present study, the 'Supervent' box design with outer dimensions $(400 \mathrm{~mm} \times 270 \mathrm{~mm} \times 300 \mathrm{~mm})$ was randomly filled with realistic pome 
fruit geometries (see Figure 1) and subjected to horizontal-airflow FAC. The mechanical properties of the 'Supervent' were obtained from Fadiji et al. (Fadiji et al., 2017). In this study, a single box was considered with the purpose of evaluating the effect of fruit shape.

\subsection{Fruit shape modelling}

The fruit shape modelling procedure is explained in detail for pome fruit geometries but is applicable to all horticultural products. Realistic apple and pear shape geometries were constructed with a validated geometrical 3D shape model generator developed by Rogge et al. (Rogge et al., 2015, 2014). In short, projection images of 65 apples (Malus $\times$ domestica Borkh, cv. 'Jonagold') and 66 pears (pyrus communis, cv. 'Conference') were acquired with non-destructive X-ray computed tomography (CT) scans made on a MicroFocus X-ray CT (AEA Tomohawk, Philips, The Netherlands) using a Philips HOMX 161 X-ray source, operating at $80 \mathrm{kV}$ and $240 \mu \mathrm{A}$. The voxel size in the resulting images ranged from 81 to 106 $\mu \mathrm{m}$. By rotating the $\mathrm{X}$-ray source with an angular increment of $0.4^{\circ}$, multiple projections were obtained that were reconstructed into 3D volumetric images using the filtered backprojection method (Zeng, 2010). After calculating the mass centre of each fruit in spherical coordinates, it was transformed into a 2D shape signature $(r)$ depending on the azimuth $(\theta)$ and elevation $(\phi)$. The 3D fruit contour was represented directly with a 2D Fourier series of the 2D shape signature. In general, a complete orthogonal family of the Fourier expansion of a function with two variables is given by (Shapiro, 1963):

$$
\begin{aligned}
r(\theta, \phi)= & \sum_{n=1}^{\infty} \sum_{m=1}^{\infty} c_{n, m} \sin \left(\frac{n \pi \theta}{a}\right) \sin \left(\frac{m \pi \phi}{b}\right) \\
& \text { with } 0<\theta<a, \text { and } 0<\phi<b
\end{aligned}
$$


where $a$ and $b$ define the domain size. Here, $a=2 \pi$ and $b=\pi$. From orthogonality arguments, the coefficients $C_{n, m}$ are expressed by:

$$
\begin{aligned}
c_{n, m}= & \frac{4}{a b} \int_{0}^{b} \int_{0}^{a} r(\theta, \phi) \sin \left(\frac{n \pi \theta}{a}\right) \sin \left(\frac{m \pi \phi}{b}\right) d \theta d \phi \\
& \text { for } n, m=1,2,3, \ldots
\end{aligned}
$$

A Fourier series expansion of the centroid distance shape signature (Kreyszig, 2010) was performed to obtain common (1D) Fourier descriptors that described the outer shape of the fruit:

$$
r\left(\phi^{\prime}\right)=\frac{a_{0}}{2}+\sum_{k=1}^{\infty}\left[a_{k} \cos \left(k \phi^{\prime}\right)+b_{k} \sin \left(k \phi^{\prime}\right)\right]
$$

where $\phi^{\prime}$ is the rotation angle and the coefficients $a_{k}$ and $b_{k}$ expressed by orthogonality:

$$
a_{k}=\frac{1}{\pi} \int_{0}^{2 \pi} r\left(\phi^{\prime}\right) \cos \left(k \phi^{\prime}\right) d \phi^{\prime} ; b_{k}=\frac{1}{\pi} \int_{0}^{2 \pi} r\left(\phi^{\prime}\right) \sin \left(k \phi^{\prime}\right) d \phi^{\prime}
$$

By scanning a large batch of fruit, an extensive dataset with statistical distributions of the shape descriptors was developed. On this dataset, the covariance decomposition algorithm (Rubinstein, 1981) used the average of each descriptor $c_{n, m}, a_{k}, b_{k}$, and the covariance between the descriptors to randomly create new sets of shape descriptors. The resulting shape descriptor sets are representative for the original distributions (Rogge et al., 2015). These new sets were then transformed back into new geometric 3D shape models, represented by point clouds. On each of the point clouds, a Non-Uniform Rational B-Spline (NURBS) surface was fitted resulting in an extensive database of more than 150 surface 
meshed bodies that also considered the biological variability of the cultivar. The model generator was coded entirely in Matlab (The MathWorks Inc., Natick, MA) and was optimised in terms of calculation time and manual interventions. A 64-bit Intel® Core2 Quad Q9650, 3.00 GHz, 8 GB RAM Windows 7 computer was used for all calculations.

\subsection{Randomised fruit stack modelling}

In a subsequent step, the gravity-driven filling of the 'Supervent' package with realistically shaped pome fruit geometries was modelled with the Discrete Element Method (DEM) in order to have a realistic but random filling pattern of the package. With DEM, the time-dependent interactions between moving discrete macroscopic visco-elastic bodies in an assembly are simulated. After calculating the contact forces and particle displacements over a discrete time step, new locations for each body are determined (Tijskens et al., 2003).

With the recently developed method of Smeets et al. (Smeets et al., 2015, 2014), DEM simulations with fruit exhibiting non-spherical curvatures such as apples and pears were performed. Instead of directly computing the contact forces, this method explicitly integrates the pressure from classical Hertz contact theory over the contact area between two bodies. However, when the contact pressure surpasses a critical value, pome fruit tissue behaves visco-elastoplastically (Van Zeebroeck, 2005). Therefore, a validated visco-elastoplastic contact force model was used to simulate colliding pome fruit (Diels et al., 2016). The forcedeformation relationship of the visco-elastoplastic model was discussed in detail by Diels et al. (2016). This model captures the visco-elastoplastic behaviour of fruit using four material properties, namely a dissipative parameter $A$ which is a measure of viscosity, the Young's modulus $E$, the Poisson's ratio $v$ and the yield pressure $p_{y}$. which is the maximal pressure before plastic deformation (i.e. bruising) occurs. The dissipative parameter in the contact force model was required to acquire reliable estimates for both the absorbed energy and force 
during collisions at small impact velocities. For Jonagold apples, these properties have already been determined by Diels et al. (Diels et al., 2016) from quasi-static and dynamic mechanical measurements $\left(A=0.0014 \mathrm{~s}, E=3.74 \mathrm{MPa}, v=0.30, p_{y}=0.63 \mathrm{MPa}\right)$. In this study, these mechanical measurements were repeated on 33 'Conference' pears, collected on January $9^{\text {th }} 2018$ from a commercial grower (Van Der Velpen; Bierbeek, Belgium). The pears were picked on August 28 2017 at Bierbeek (Belgium) and stored for 4 months under controlled atmosphere $\left(3 \% \mathrm{O}_{2}\right.$, below $\left.0.7 \% \mathrm{CO}_{2},-0.5{ }^{\circ} \mathrm{C}\right)$. The Young's modulus was derived from 20 quasi-static measurements on half pears using a Universal test machine with a $500 \mathrm{~N}$ load cell (Type LS1 Material Tester, LLoyd materials testing, West Sussex, UK). Assuming a Poisson's ratio of 0.30 , a Young's modulus of $2.54 \pm 0.28 \mathrm{MPa}$ was found by fitting Hertz's law to the force-deformation curves (average $\mathrm{R}^{2}=0.997$ ). For the dynamic experiment, in total 45 half pears were impacted with the pendulum device described in Diels et al. (Diels et al., 2016) whereby nine different impact energy levels were used. The parameters $A$ and $p_{y}$ were derived by fitting the visco-elastoplastic contact force model to the impact data and were found to be equal to $0.0033 \mathrm{~s}$ and $0.7500 \mathrm{MPa}$, respectively. For more information about the mechanical experiments and the subsequent parameter estimation the reader is referred to the work of Diels et al. (Diels et al., 2016).

In this study, the DEM simulation started from an assembly of apples and pears, which were arbitrarily selected from the database developed in Section 2.2. These bodies were given an initial randomised orientation to simulate a realistic filling operation. To obtain the position and velocity of each apple and pear at each time step $(2 \mu \mathrm{s})$, the forces acting on the colliding apples (gravity and collision forces) were summed and Newton's and Euler's equations of motion (for translation and rotation, respectively) were integrated over the time 
step (Tijskens et al., 2003; Van Staeyen et al., 2003). This resulted in a randomised filling pattern of apple and pear fruit (Figure 1).

Next, the Cartesian coordinates of the mass centre and the hydraulic diameter of each individual apple were calculated and used to reconstruct the apple filling with regular spheres. However, it should be noted that this spherical filling pattern is not a mechanically correct end result of a gravity-driven filling operation of the 'Supervent' with regular spheres. Since pears are non-spherical bodies, no spherical filling pattern was reconstructed for this filling pattern because it would lead to either overlapping or 'floating' bodies. The average hydraulic diameter of the apples and pears were $73.1 \pm 4.2$ and $68.3 \pm 5.2 \mathrm{~mm}$, respectively. By matching the total volume between the two filling patterns (minor mismatch of $1.57 \% \pm 0.47$ $\%$ ) and, thus, the porosity, a spherical representation of the apple filling pattern with spheres having an equivalent diameter equal to the respective apples was created. On the top and bottom side of a single apple, the petiole and calyx ends create concave fruit shapes. The average surface-to-volume ratio $\left(\mathrm{m}^{-1}\right)$ was $98.77 \pm 7.29,84.48 \pm 1.02$ and $77.50 \pm 0.40$ for the pear, apple and spherical fillings, respectively. Consequently, the total exchange area for heat at the fruit surfaces of the apple filling pattern was $8.59 \% \pm 1.57 \%$ larger than the spherical representation, although the volume and mass of fruit was very similar between both. With the procedure explained above, three randomised filling patterns of 69,71 and 73 apples in the 'Supervent' and their spherical representations were generated. In addition, one pear filling with 89 pears in the 'Supervent' was generated. Figure 1 shows the geometries of the 'Supervent' box design, the pear filling pattern and one filling pattern of apples with its spherical equivalent. 


\subsection{CFD model}

A CFD model was generated that resolved the heat and mass transport in the fruit and the airspaces between the different fruit during FAC. In Figure 2, the computational domain and the implemented boundary conditions (BCs) are depicted, which are used to simulate airflow and heat transfer for a horizontal FAC operation of packed apples and pears in the 'Supervent'. Steady-state simulations of the airflow and heat transfer of the filling patterns were performed, using a constant fruit surface temperature of $20{ }^{\circ} \mathrm{C}$ and airflow entering the computational domain at $0{ }^{\circ} \mathrm{C}$. These simulations are used to identify distributions of air velocity, pressure loss over the packages and the convective heat transfer coefficient distribution on the fruit surfaces. For one filling pattern, a transient heat transfer simulation was performed to evaluate the cooling performance via the time-dependent changes of the fruits' core temperature. The fruit and the 'Supervent' package were modelled as solid domains while the air was modelled as a fluid domain. The thermo-physical properties of the apple and pear fruit, 'Supervent' package and air (ASHRAE, 2013; Ho et al., 2010; Lisowa et al., 2002; Nahor et al., 2005) were kept constant throughout the simulation due to the relatively small temperature range (Ferrua and Singh, 2009) and are listed in Table 1 . The inlet was modelled with a uniform velocity BC with medium turbulence intensity (5\%). Three realistic airflow rates in the range of what is commonly used in the precooling industry (ASHRAE, 2010; Brosnan and Sun, 2001; de Castro et al., 2004; Thompson et al., 2008) were assessed, namely 1, 2 and $3 \mathrm{~L} \mathrm{~s}^{-1} \mathrm{~kg}^{-1}$ (low, medium and high flow rate). For comparative purpose, the medium flow rate for the pear filling was $1.5 \mathrm{~L} \mathrm{~s}^{-1} \mathrm{~kg}^{-1}$ to match the flow rate of other two fillings. The resulting Reynolds numbers were calculated by Eq. 5: 


$$
R e_{D}=\frac{U_{s} d_{p}}{\mu}
$$

with $U_{s}$ the superficial velocity presented in Table $2\left[\mathrm{~m} \mathrm{~s}^{-1}\right], \mu$ the kinematic viscosity of air $\left[1.46 \cdot 10^{-5} \mathrm{~kg} \mathrm{~m}^{-1} \mathrm{~s}^{-1}\right]$ (Incropera and De Witt, 1990). The particle diameter, $d_{p}[\mathrm{~m}]$, of the apples/spheres was calculated by taking the average hydraulic diameter of all fruit in the three fillings $(72.0 \pm 4.2 \mathrm{~mm})$. For the non-spherical pear fruit, $d_{p}$ is defined as (Gaskell, 2012):

$$
d_{p}=\left(\frac{6 V}{\pi}\right)^{\frac{1}{3}}
$$

with $V$ the particle volume $\left[\mathrm{m}^{3}\right](68.3 \pm 5.2 \mathrm{~mm})$. The outlet of the air domain was modelled as a zero static pressure BC. By modelling all sides of the computational domain as symmetry planes, the model actually represents a package in a stack of multiple boxes. The surfaces of the 'Supervent' and the fruit were modelled as no-slip walls with zero roughness. Buoyancy, radiation, the heat generated by respiration, the moisture evaporation at the surface of the fruit and thus, the latent heat removal were assumed to have a negligible effect in the FAC process (Defraeye et al., 2013a; Gowda et al., 1997; Redding et al., 2016; Tanner et al., 2002). Standard Reynolds-averaged Navier-Stokes (RANS) equations were solved in combination with the shear stress transport $k-\omega$ turbulence model (SST $k$ - $\omega$ model; [30-32]). The SST $k$ - $\omega$ model used automatic wall functions to automatically switch from a low-Re formulation to wall functions (ANSYS, 2014), which were used to predict the heat exchange at the interfaces between the fruit and air (Defraeye et al., 2013b). The flow quantities in the boundary-layer region were modelled with a fine grid resolution to ensure 
low $\mathrm{y}^{+}$values. Via an iterative process, the $\mathrm{y}^{+}$values of the apple filling pattern and its corresponding spherical representation were closely matched. For the low, medium and high flow rate, the surface averaged $\mathrm{y}^{+}$-values for the three different filling patterns were $5.96 \pm$ $0.10,9.93 \pm 0.02$ and $14.33 \pm 0.06$. For the pear filling, the surface averaged $\mathrm{y}^{+}$values were $7.78,10.32$ and 19.09 respectively.

\subsection{Solution procedure}

In this study, the finite volume technique was used in all simulations to solve the transport equations of the model using the commercial software ANSYS CFX 17.2. A hybrid grid with tetrahedral and hexahedral mesh cells was used to discretise all domains. Figure 1 shows the mesh for the apple and pear filling. Richardson extrapolation (Celik et al., 2008; Franke et al., 2007; Roache, 1994) was used in a mesh sensitivity analysis to calculate the spatial discretization error. With an average total of $2.78 \times 10^{6}, 2.91 \times 10^{6} \pm 1.14 \times 10^{6}$ and $3.54 \times 10^{6} \pm 3.07 \times 10^{5}$ mesh elements for the pear model, the three apple model and the spherical representations, respectively, the discretization error was maximally $1.5 \%$ for the pressure drop over the 'Supervent' package and for the average heat flux from the apples and pears. The mass imbalance, and the temperature, velocity and turbulent kinetic energy at specific locations in the flow field were monitored to assess the iterative convergence. To speed up the calculations, the mass and momentum equations were solved decoupled from the heat transfer equations. A converged steady-state solution for the airflow was used as an initial condition for obtaining a solution of the unsteady heat transfer equations. Based on a temporal sensitivity analysis, all transient simulations were run for $20 \mathrm{~h}$ with a time step of 2 min and 25 iterations each. The advection scheme used a high-resolution spatial differencing scheme and the transient scheme the second-order backward Euler method, which is an implicit time-stepping scheme that is second-order accurate (ANSYS, 2016). A 64-bit Intel® 
Xeon® CPU E5-2630, $2.30 \mathrm{GHz}, 64 \mathrm{~GB}$ RAM, Windows 7 PC was used to perform all calculations which took on average $12 \mathrm{~h}$.

\section{Results}

\subsection{Aerodynamic resistance characteristics and airflow distribution}

The calculated pressure drop over the package, filled with fruit, as a function of the flow rate, is shown in Figure 3 for pears, apples and their equivalent sphere filling pattern. The flow rate is expressed in superficial velocity $\left(\mathrm{m} \mathrm{s}^{-1}\right)$ and specific flow rate per fruit mass (L $\left.\mathrm{s}^{-1} \mathrm{~kg}^{-1}\right)$. In addition, the average aerodynamic resistance curves of the pear, apple and equivalent sphere fillings are plotted in Figure 3, to which a quadratic relation is fitted (see Table 3). This relation is described by the Darcy-Forchheimer equation:

$$
\Delta P=\xi_{1} U_{s}^{2}+\xi_{2} U_{s}
$$

where $\xi_{1}$ and $\xi_{2}$ are pressure loss coefficients. At high speeds, the pressure drop over the package is governed by the Forchheimer term that accounts for inertial effects (first term of Eq. 7) while at very low flow rates, indicated by $R e_{p}<1$, viscous effects become dominant (second term of Eq. 7) (van der Sman, 2002; Verboven et al., 2006). The pressure drop calculated in this study corresponded well with observations of Defraeye et al. $(2015,2014)$.

The total mass of pear fruit in the 'Supervent' was $5.39 \%$ higher than that of apple fruit. Consequently, the average pressure drop of apples and pears was different when expressed against specific airflow rate $\left(\mathrm{L} \mathrm{s}^{-1} \mathrm{~kg}^{-1}\right)$, while it was the same for the same superficial velocity. The individual apple filling patterns differed noticeably in pressure drop, especially at high flow rate (378 $\pm 51 \mathrm{~Pa}$ and $369 \pm 32 \mathrm{~Pa}$ for apple and spheres, respectively). However, 
the difference between the apple and sphere filling pattern was negligible. At medium flow rate, the average pressure drop over the fruit-filled 'Supervent' box was $154.3 \pm 14.7 \mathrm{~Pa}$ for the apple filling and 150.2 \pm 9.3 Pa for the sphere filling. The pressure drop over the apples and spheres (without packaging) was only $2.1 \pm 0.8 \mathrm{~Pa}$ and $1.7 \pm 0.5 \mathrm{~Pa}$, respectively. Thus, the main contributor to the overall pressure drop was the package design and not the fruit. In any case, however, the different apple filling patterns seem to affect the pressure drop also significantly, especially if the vent holes are (partially) blocked by the filling.

The airflow pattern inside the 'Supervent' of one filling pattern with 71 apple fruit at medium flow rate is depicted in Figure 4. In all cases, a high degree of heterogeneity in local air velocity was observed. The vent holes are located on the bottom and top edges of the 'Supervent' box design, resulting in an airflow that can easily bypass the fruit in the centre of the box by traveling along the bottom and top walls. Furthermore, in the middle of the fruit stack, very low air velocities were observed, a phenomenon that was more pronounced in the case with real fruit shapes.

\subsection{Cooling heterogeneity}

Convective heat transfer coefficients are well suited to identify local cooling heterogeneity within fruit stacks (Berry et al., 2017). In this study, the convective heat transfer coefficient $\left(h\left[\mathrm{~W} \mathrm{~m} \mathrm{~m}^{-2} \mathrm{~K}^{-1}\right]\right)$ was calculated as the steady-state local surface heat flux divided by the difference between the constant inlet and initial fruit surface temperatures. In general, high cooling rates are associated with high values of $h$ while the degree of cooling heterogeneity is shown by the differences in $h$ distributed throughout the filling pattern. Figure 5 illustrates a surface contour plot of $h$ for the apple filling, its spherical counterpart and the pear filling at medium flow rate. Large differences in $h$ between each filling pattern were apparent. For this filling, the surface averaged $h$ of apples $\left(31.9 \mathrm{~W} \mathrm{~m}^{-2} \mathrm{~K}^{-1}\right)$ was about 
$6.24 \%$ smaller than that of its spherical equivalent $\left(34.0 \mathrm{~W} \mathrm{~m}^{-2} \mathrm{~K}^{-1}\right)$. For the case of the pear filling, the surface averaged $h$-value $\left(27.8 \mathrm{~W} \mathrm{~m}^{-2} \mathrm{~K}^{-1}\right)$ was considerably lower at the same airflow rate and its distribution was more heterogeneous than was the case for the apples and spheres. Figure 6 shows the surface averaged $h$-values on the entire fruit surface, expressed as a function of $\operatorname{Re}_{D}$ (Eq. 5). The surface averaged $h$-values per flow rate are presented in Table 4. As a reference, theoretical correlations of gas flow through a packed bed of uniform solid particles were also presented in Figure 6. The correlations are presented by Eq. 8 and 9:

$$
\begin{aligned}
& \varepsilon j_{H}=2.06 \operatorname{Re}_{D}^{-0.575} \\
& j_{H}=\frac{h}{\rho U_{s} C_{p}} \operatorname{Pr}^{0.667}
\end{aligned}
$$

where $j_{H}$ the Colburn $j$ factor [-], $h$ the theoretical convective heat transfer coefficient $\left[\mathrm{W} \mathrm{m} \mathrm{m}^{-2} \mathrm{~K}^{-1}\right], C_{p}$ the specific heat capacity of air $\left[1004.4 \mathrm{~J} \mathrm{~kg}^{-1} \mathrm{~K}^{-1}\right], \operatorname{Pr}$ the Prandtl number (= 0.7). The pears were assumed as a packed bed of uniformly sized cylinders and the righthand side of Eq. 8 was multiplied with a shape correction factor of 0.79 . In the correlation equation, the Reynolds number is defined in terms of the hydraulic diameter and the upstream velocity that would exist in the empty channel without the packing (Eq. 5; Incropera and De Witt, 1990). The correlation equation was developed for a packed bed of uniformly shaped particles that were not enclosed by an obstruction in the airstream. As can be seen from Figure 5 , the presence of the package design and the irregular shapes and sizes of the particles resulted in regions of high $h$-values where local air velocities were high. This explains the observed differences between the simulation and correlation results. Between the different fruit filling patterns, the differences are clearly apparent. By increasing the airflow rate, the 
differences in surface-averaged convective transfer coefficients between all cases differed ever more. The large difference between the pear and apple/sphere filling pattern can be explained by the lower porosity in the pear box $(47.87 \%$ compared to $51.21 \% \pm 1.29 \%)$ and the larger surface-to-volume ratio $\left(98.77 \pm 7.29 \mathrm{~m}^{-1}\right.$ for pear compared to $84.48 \pm 1.02$ $\mathrm{m}^{-1}$ and $77.50 \pm 0.40 \mathrm{~m}^{-1}$ for apple and sphere, respectively).

The relative frequency distributions of the normalised $h$-value provide a means to quantify the cooling heterogeneity in the box. The normalisation was done by dividing the local $h$-value with the surface-averaged $h$-value of all the fruit surfaces. In general, fruit with a normalised $h$-value much smaller than 1 will cool, on average, slower than the average. A sharp peak around this $h$-value also signifies that most of the local $h$-values lie relatively close to the surface-averaged $h$-value. Therefore, the fruit in the box cool more homogeneous if the relative frequency has a positive kurtosis around the normalised $h$-value of 1 . Figure 7 presents the average relative frequency distributions of the normalised $h$-value of the three apple filling patterns, its spherical representations and the pear filling pattern, for the three airflow rates. For all three airflow rates, the spheres have a distribution with a higher peak closer to the value of 1 . The apple fillings show a more heterogeneous distribution, with a peak considerably lower than 1 ; an observation that was even more pronounced for the pear filling. However, all distributions displayed a positive skewness. Figure 5 demonstrates that regions in the fruit filling with relatively large $h$-values correspond well with the regions of high air velocities, namely located close to the vent holes and in the top region of the box. Fruit located in front of the vent holes have much larger $h$-values than the average, accounting for the positive skewness in the relative frequency distributions. For the pear filling, the peak of the relative frequency distribution was much lower and flatter. Thus, the pear filling showed a more heterogeneous cooling process compared to the other fillings. 


\subsection{Fruit cooling behaviour}

Figure 8 shows time-temperature profiles calculated in the core of all apple fruit in the 'Supervent' package and the volume-averaged time-temperature profile of the entire fruit stack (71 fruit) of both the apple filling and its spherical representation at medium flow rate. The seven-eighths cooling time (SECT) is the time it takes to cool the produce down from its initial temperature to one-eighth of the initial temperature (Brosnan and Sun, 2001). Based on the volume-averaged temperature profiles, the SECT of the entire fruit filling deviated only 6 min between apples and spheres. To some extent, the difference in surface averaged $h$-value (6.24 \% lower for apple) was, therefore, partially compensated by the difference in total fruit surface area (9.26\% higher for apple), resulting in similar overall cooling rates. Since the total volume of the two fruit filling patterns were matched, the difference in average cooling rate between the two patterns was negligible.

However, larger differences were observed with respect to the core temperatures of the individual fruit. Distributions of the SECT of all fruit for the apple and spherical representation are shown in Figure 9. In total, $61 \%$ of all fruit in the apple filling pattern cooled faster than their spherical equivalent. On average, there was a difference of $18 \pm 15$ min difference in SECT between the two filling patterns with the smallest and largest difference being 1 and $66 \mathrm{~min}$. The faster cooling of realistically shaped apples could be explained by the presence of the calyx and petiole regions (i.e., the concave surfaces on the top and bottom of the apple). They lead to a reduced effective distance between the surface and core, resulting in a smaller SECT than its spherical counterpart. In addition, the apples had a larger surface-to-volume ratio compared to the spheres. Clearly, local cooling behaviour of packed non-spherical fruit such as pears will be even more variable. For these products, the realistic shape should be taken into account. 


\section{Discussion}

Cooling uniformity is one of the most important evaluation criteria for novel package designs. This criterion is not only influenced by the package design itself (Berry et al., 2016) but also by the produce shape, as was shown in this study. Since the computational load of the realistic shapes was of the same order as the simplified geometries, more accuracy in the cooling behaviour can be attained without making concessions on computing time. Thus, for future package design model simulations, it becomes a requirement to incorporate the realistic fruit shapes with their biological variability. A simplified product shape approach is sufficient for design studies where only the average cooling behaviour is required.

\section{Conclusions}

This study described a novel methodology for design and engineering of agrofood cooling processes by using realistic 3D shape models of agrofood products, stacked in randomized configurations. Forced-air cooling of apple and pear fruit in fibreboard boxes was considered. The cooling performance of boxes with apple fruit shapes was compared with simplified representations using spheres with the same equivalent hydraulic diameter. On average, there was no significant difference in the average aerodynamic resistance and product cooling rate of the two approaches. If design studies would target dimensioning of equipment such as fans, a simplified product shape approach would thus be sufficient.

However, in cooling of agrofood products, not only the average cooling behaviour but also the cooling uniformity between different products is of concern, as this will have a direct impact on quality differences between individual fruit. Our study showed that considerable differences in pressure drop exist between different randomized fillings. Different preferential airflow paths are induced in stacked boxes, leading to warmer and colder 
locations inside the box. Within boxes filled with apples, we have shown that the variability of convective heat transfer coefficients is somewhat larger on realistic shapes than on spheres. In addition, a lower surface heat transfer occurred on the realistic apple shapes. However, the concave apple surfaces caused an increase in cooling rate of the apple cores. As a combined result, cooling uniformity was overestimated by the spherical geometries, with $25 \%$ longer cooling times predicted for the slowest cooling fruit in the box.

The results of the pear filling showed a much larger variability in convective heat transfer coefficients. In this case, incorporating the actual fruit shape will be necessary to capture all effects on cooling behaviour. For fruit shapes that are more deviating from spheres, the observed effects of cooling uniformity and local cooling rates will thus be more pronounced. The presented novel and more versatile methodology can now be readily applied without shape simplification to improve package designs of different types of horticultural produce.

\section{Acknowledgements}

This work was supported by the Flanders Innovation \& Entrepreneurship (project VLAIO 120745). Thijs Defraeye acknowledges the support of the World Food System Center (WFSC) of ETH Zürich (www.worldfoodsystem.ethz.ch) and the Swiss National Science Foundation SNSF (project 200021_169372).

\section{References}

Alvarez, G., Flick, D., 1999. Analysis of heterogeneous cooling of agricultural products inside bins: Part II: thermal study. J. Food Eng. 39, 239-245.

ANSYS, 2016. ANSYS CFX 17.2 Reference Guide. ANSYS, Inc., Canonsburg, PA, USA. ANSYS, 2014. ANSYS Fluent User's Guide 15.0.

ASHRAE, 2013. ASHRAE: Handbook - Fundamentals.

ASHRAE, 2010. ASHRAE Handbook: Refrigeration, SI Edition. ed. American Society of Heating, Refrigerating and Air-Conditioning Engineers, Atlanta.

Berry, T., Defraeye, T., Nicolai, B.M., Opara, U.L., 2016. Multiparameter analysis of cooling efficiency of ventilated fruit cartons using CFD: impact of vent hole design 
and internal packaging. Food Bioprocess Technol. doi:10.1007/s11947-016-1733-y

Berry, T., Fadiji, T.S., Defraeye, T., Opara, U.L., 2017. The role of horticultural carton vent hole design on cooling efficiency and compression strength: A multi-parameter approach. Postharvest Biol. Technol. 124, 62-74.

doi:10.1016/j.postharvbio.2016.10.005

Brosnan, T., Sun, D.W., 2001. Precooling techniques and applications for horticultural products - a review. Int. J. Refrig. 24, 154-170.

Celik, I.B., Ghia, U., Roache, P.J., Freitas, C.J., 2008. Procedure for estimation and reporting of uncertainty due to discretization in CFD applications. J. Fluids Eng. 130, 4. doi:10.1115/1.2960953

Clement, J., Novas, N., Gazquez, J.A., Manzano-Agugliaro, F., 2013. An active contour computer algorithm for the classification of cucumbers. Comput. Electron. Agric. 92, 75-81. doi:10.1016/j.compag.2013.01.006

de Castro, L.R., Vigneault, C., de Cortez, L.A.B., 2004. Effect of container opening area on air distribution during precooling of horticultural produce. Trans. ASAE 47, 2033 2038.

Defraeye, T., Cronjé, P., Verboven, P., Opara, U.L., Nicolai, B., 2015. Exploring ambient loading of citrus fruit into reefer containers for cooling during marine transport using computational fluid dynamics. Postharvest Biol. Technol. 108, 91-101. doi:10.1016/j.postharvbio.2015.06.004

Defraeye, T., Herremans, E., Verboven, P., Carmeliet, J., Nicolai, B., 2012. Convective heat and mass exchange at surfaces of horticultural products: A microscale CFD modelling approach. Agric. For. Meteorol. 162-163, 71-84. doi:10.1016/j.agrformet.2012.04.010

Defraeye, T., Lambrecht, R., Delele, M.A., Tsige, A.A., Opara, U.L., Cronjé, P., Verboven, P., Nicolai, B., 2014. Forced-convective cooling of citrus fruit: Cooling conditions and energy consumption in relation to package design. J. Food Eng. 121, 118-127. doi:10.1016/j.jfoodeng.2013.08.021

Defraeye, T., Lambrecht, R., Tsige, A.A., Delele, M.A., Opara, U.L., Cronjé, P., Verboven, P., Nicolai, B., 2013a. Forced-convective cooling of citrus fruit: Package design. J. Food Eng. 118, 8-18. doi:10.1016/j.jfoodeng.2013.03.026

Defraeye, T., Verboven, P., Nicolai, B., 2013b. CFD modelling of flow and scalar exchange of spherical food products: Turbulence and boundary-layer modelling. J. Food Eng. 114, 495-504. doi:10.1016/j.jfoodeng.2012.09.003

Dehghannya, J., Ngadi, M., Vigneault, C., 2012. Transport phenomena modelling during produce cooling for optimal package design: Thermal sensitivity analysis. Biosyst. Eng. 111, 315-324. doi:10.1016/j.biosystemseng.2012.01.001

Delele, M.A., Ngcobo, M.E.K., Getahun, S.T., Chen, L., Mellmann, J., Opara, U.L., 2013. Studying airflow and heat transfer characteristics of a horticultural produce packaging system using a 3-D CFD model. Part II: Effect of package design. Postharvest Biol. Technol. 86, 546-555. doi:10.1016/j.postharvbio.2013.08.015

Denys, S., Pieters, J.G., Dewettinck, K., 2003. Combined CFD and experimental approach for determination of the surface heat transfer coefficient during thermal processing of eggs. J. Food Sci. 68, 943-951. doi:10.1111/j.1365-2621.2003.tb08269.x

Diels, E., Smeets, B., Odenthal, T., Keresztes, J., Vanmaercke, S., Verboven, P., Nicolaïa, B., Saeys, W., Ramon, H., 2016. Development of a visco-elastoplastic contact force model for arbitrary rounded bodies and determining its contact parameters for apples. 
Postharvest Biol. Technol. 120, 157-166. doi:10.1017/CBO9781107415324.004

Fadiji, T., Berry, T., Coetzee, C., Opara, L., 2017. Investigating the mechanical properties of paperboard packaging material for handling fresh produce under different environmental conditions : experimental analysis and finite element modelling. J. Appl. Packag. Res. 9, 20-34.

Ferrua, M.J., Singh, R.P., 2011. Improved airflow method and packaging system for forced-air cooling of strawberries. Int. J. Refrig. 34, 1162-1173. doi:10.1016/j.ijrefrig.2011.01.018

Ferrua, M.J., Singh, R.P., 2009. Modeling the forced-air cooling process of fresh strawberry packages, Part I: Numerical model. Int. J. Refrig. 32, 335-348. doi:10.1016/j.ijrefrig.2008.04.010

Franke, J., Hellsten, A., Schlünzen, H., Carissimo, B., 2007. Best practice guidelines for the CFD simulation of flows in the urban environment. COST Action 732 Qual. Assur. Improv. microscale Meteorol. Model. Hamburg, Ger.

Gaskell, D.R., 2012. An introduction to transport phenomena in materials engineering, 2nd ed. Momentum Press, LLC, New York, USA. doi:10.5643/9781606503577

Goñi, S.M., Purlis, E., 2010. Geometric modelling of heterogeneous and complex foods. J. Food Eng. 97, 547-554. doi:10.1016/j.jfoodeng.2009.11.017

Gowda, B.S., Narasimham, G.S.V.L., Murthy, M.V.K., 1997. Forced-air precooling of spherical foods in bulk: A parametric study. Int. J. Heat Fluid Flow 18, 613-624. doi:10.1016/S0142-727X(97)00028-3

Han, J.W., Zhao, C.J., Yang, X.T., Qian, J.P., Fan, B.L., 2015. Computational modeling of airflow and heat transfer in a vented box during cooling: Optimal package design. Appl. Therm. Eng. 91, 883-893. doi:10.1016/j.applthermaleng.2015.08.060

Ho, Q.T., Rogge, S., Verboven, P., Verlinden, B.E., Nicolaï, B.M., 2016. Stochastic modelling for virtual engineering of controlled atmosphere storage of fruit. J. Food Eng. 176, 77-87. doi:10.1016/j.jfoodeng.2015.07.003

Ho, S.H., Rahman, M.M., Sunol, A.K., 2010. Modelling of the thermal response of a multi-tray food self-heating unit 68, 123-134. doi:10.2495/HT100111

Incropera, F.P., De Witt, D.P., 1990. Fundamentals of heat and mass transfer, Third Edit. ed. Jonn Wiley \& Sons, Inc.

Jackson, J.E., 2003. The biology of apples and pears - Biology of horticultural crops. Cambridge University Press, Cambridge, United Kingdom.

Kreyszig, E., 2010. Advanced Engineering Mathematics, 10th Editi. ed. John Wiley and Sons.

Launder, B.E., Spalding, D.B., 1974. The numerical computation of turbulent flows. Comput. Methods Appl. Mech. Eng. 3, 269-289. doi:10.1016/0045-7825(74)90029-2

Lisowa, H., Wujec, M., Lis, T., 2002. Influence of temperature and variety on the thermal properties of apples. Int. agrophysics 16, 43-52.

Menter, F.R., 1994. Two-equation eddy-viscosity turbulence models for engineering applications. AIAA J. 32, 1598-1605.

Nahor, H.B., Hoang, M.L., Verboven, P., Baelmans, M., Nicolaï, B.M., 2005. CFD model of the airflow, heat and mass transfer in cool stores. Int. J. Refrig. 28, 368-380. doi:10.1016/j.ijrefrig.2004.08.014

O’Sullivan, J., Ferrua, M.J., Love, R., Verboven, P., Nicolaï, B., East, A., 2016. Modelling the forced-air cooling mechanisms and performance of polylined horticultural 
produce. Postharvest Biol. Technol. 120, 23-35.

doi:10.1016/j.postharvbio.2016.05.008

Olatunji, J.R., Love, R.J., Shim, Y.M., Ferrua, M.J., East, A.R., 2017. Quantifying and visualising variation in batch operations: A new heterogeneity index. J. Food Eng. 196, 81-93. doi:10.1016/j.jfoodeng.2016.10.004

Redding, G.P., Yang, A., Shim, Y.M., Olatunji, J., East, A., 2016. A review of the use and design of produce simulators for horticultural forced-air cooling studies. J. Food Eng. 190, 80-93. doi:10.1016/j.jfoodeng.2016.06.014

Roache, P.J., 1994. Perspective: a method for uniform reporting of grid refinement studies. J. Fluids Eng. 116, 405-413.

Rogge, S., Beyene, S.D., Herremans, E., Hertog, M.L., Defraeye, T., Verboven, P., Nicolai, B., 2014. A geometrical model generator for quasi-axisymmetric biological products. Food Bioprocess Technol. 7, 1783-1792.

Rogge, S., Defraeye, T., Herremans, E., Verboven, P., Nicolaï, B.M., 2015. A 3D contour based geometrical model generator for complex-shaped horticultural products. J. Food Eng. 157, 24-32. doi:10.1016/j.jfoodeng.2015.02.006

Rubinstein, R.Y., 1981. Simulation and the Monte Carlo Method. John Wiley and Sons, New York, USA.

Shapiro, V.L., 1963. Fourier series in several variables. Bull. Am. Math. Soc. 70, 47-93.

Smeets, B., Odenthal, T., Keresztes, J., Vanmaercke, S., Van Liedekerke, P., Tijskens, E., Saeys, W., Van Oosterwyck, H., Ramon, H., 2014. Modeling contact interactions between triangulated rounded bodies for the discrete element method. Comput. Methods Appl. Mech. Eng. 277, 219-238. doi:10.1016/j.cma.2014.04.017

Smeets, B., Odenthal, T., Vanmaercke, S., Ramon, H., 2015. Polygon-based contact description for modeling arbitrary polyhedra in the Discrete Element Method. Comput. Methods Appl. Mech. Eng. 290, 277-289. doi:10.1016/j.cma.2015.03.004

Taiz, L., Zeiger, E., Møller, I.M., Murphy, A., 2015. Plant physiology and development, 6th ed. Sinauer Associates, Inc., Sunderland, Massachusetts U.S.A.

Tanner, D.J., Cleland, A.C., Opara, U.L., Robertson, T.R., 2002. A generalised mathematical modelling methodology for design of horticultural food packages exposed to refrigerated conditions: part 1, formulation. Int. J. Refrig. 25, 33-42. doi:10.1016/S0140-7007(01)00019-6

Thompson, J.F., Mitchell, F.G., Rumsey, T.R., Kasmire, R.F., Crisosto, C.H., 2008. Commercial cooling of fruits, vegetables, and flowers. University of California, California.

Tijskens, E., Ramon, H., De Baerdemaeker, J., 2003. Discrete element modelling for process simulation in agriculture. J. Sound Vib. 266, 493-514. doi:10.1016/S0022460X(03)00581-9

Tutar, M., Erdogdu, F., Toka, B., 2009. Computational modeling of airflow patterns and heat transfer prediction through stacked layers' products in a vented box during cooling. Int. J. Refrig. 32, 295-306. doi:10.1016/j.ijrefrig.2008.05.003

Uyar, R., Erdoğdu, F., 2009. Potential use of 3-dimensional scanners for food process modeling. J. Food Eng. 93, 337-343. doi:10.1016/j.jfoodeng.2009.01.034

van der Sman, R.G.., 2002. Prediction of airflow through a vented box by the DarcyForchheimer equation. J. Food Eng. 55, 49-57. doi:10.1016/S0260-8774(01)00241-2

Van Eck, J.W., Van Der Heijden, G.W.A.M., Polder, G., 1998. Accurate measurement of 
size and shape of cucumber fruits with image analysis. J. Agric. Eng. Res. 70, 335343. doi:10.1006/jaer.1998.0285

Van Staeyen, K., Tijskens, E., Ramon, H., 2003. A multibody dynamic approach for colliding particles. J. Sound Vib. 266, 481-491. doi:10.1016/S0022-460X(03)00580-7

Van Zeebroeck, M., 2005. The discrete element method (DEM) to simulate fruit impact damage during transport and handling the discrete element method (DEM) to simulate fruit impact damage during transport and handling. Phd thesis, KU Leuven 258.

Verboven, P., Flick, D., Nicolaï, B.M., Alvarez, G., 2006. Modelling transport phenomena in refrigerated food bulks, packages and stacks: basics and advances. Int. J. Refrig. 29, 985-997. doi:10.1016/j.ijrefrig.2005.12.010

Zeng, G.L., 2010. Medical image reconstruction: a conceptual tutorial. Higher Education Press, Utah, USA. 
Table 1: Thermophysical properties of air, Conference pear, Jonagold apple and the Supervent box

\begin{tabular}{lcccc}
\hline \multicolumn{1}{c}{$\mathbf{1}$} & $\begin{array}{r}\text { Density } \\
\left(\mathrm{kg} \mathrm{m}^{-3}\right)\end{array}$ & $\begin{array}{c}\text { Specific heat capacity } \\
\left(\mathrm{J} \mathrm{kg}^{-1} \mathrm{~K}^{-1}\right)\end{array}$ & $\begin{array}{c}\text { Thermal conductivity } \\
\left(\mathrm{W} \mathrm{m}^{-1} \mathrm{~K}^{-1}\right)\end{array}$ & Reference \\
\hline Air & 1.185 & 1004 & 0.024 & (ASHRAE, 2013) \\
Pear & 1000 & 3800 & 0.52 & (Nahor et al., 2005) \\
Apple & 800 & 3455 & 0.397 & (Lisowa et al., 2002) \\
Supervent & 145 & 1338 & 0.064 & (Ho et al., 2010) \\
\hline
\end{tabular}

Table 2: The flow rate in $\mathrm{L} \mathrm{s}^{-1} \mathrm{~kg}^{-1}$ and superficial velocity in $\mathrm{m} \mathrm{s}^{-1}$ for the apple, sphere and pear filling patterns.

\begin{tabular}{lccccccccc}
\hline & \multicolumn{3}{c}{ Apple } & \multicolumn{3}{c}{ Sphere } & \multicolumn{3}{c}{ Pear } \\
\cline { 2 - 10 } $\begin{array}{l}\text { Flow rate } \\
\left(\mathrm{L} \mathrm{s}^{-1} \mathrm{~kg}^{-1}\right)\end{array}$ & 1.0 & 2.0 & 3.0 & 1.0 & 2.0 & 3.0 & 1.0 & 1.5 & 3.0 \\
\hline Pattern 1 $\left(\mathrm{m} \mathrm{s}^{-1}\right)$ & 0.146 & 0.276 & 0.438 & 0.145 & 0.276 & 0.436 & 0.192 & 0.276 & 0.575 \\
Pattern 2 $\left(\mathrm{m} \mathrm{s}^{-1}\right)$ & 0.139 & 0.276 & 0.416 & 0.141 & 0.276 & 0.424 & - & - & - \\
Pattern 3 $\left(\mathrm{m} \mathrm{s}^{-1}\right)$ & 0.147 & 0.276 & 0.441 & 0.147 & 0.276 & 0.439 & - & - & - \\
\hline
\end{tabular}

Table 3: Average polynomial approximation of the pressure drop characteristics for the filling patterns of apples, spheres and pears as a function of the flow rate through the computational domain expressed in $\mathrm{m} \mathrm{s}^{-1}$ and $\mathrm{L} \mathrm{s}^{-1} \mathrm{~kg}^{-1}$.

\begin{tabular}{lll}
\hline Filling pattern & Superficial air velocity in $\mathrm{m} \mathrm{s}^{-1}$ & Superficial air velocity in $\mathrm{L} \mathrm{s}^{-1} \mathrm{~kg}^{-1}$ \\
\hline Apple & $\Delta P=2046 U_{s}^{2}-6 U_{s}$ & $\Delta P=42.3 U_{s}^{2}-1.1 U_{s}$ \\
Sphere & $\Delta P=1978 U_{s}^{2}-4 U_{s}$ & $\Delta P=40.5 U_{s}^{2}-0.6 U_{s}$ \\
Pear & $\Delta P=2053 U_{s}^{2}-5 U_{s}$ & $\Delta P=75.3 U_{s}^{2}-1.0 U_{s}$ \\
\hline
\end{tabular}

Table 4: Surface averaged convective heat transfer coefficient, with standard error ( 3 filling patterns) for three different airflow rates for the filling patterns of apples, spheres and pears

\begin{tabular}{lccc}
\hline Filling pattern & Low flow rate & Medium flow rate & High flow rate \\
\hline Apple & $29.7 \pm 2.7$ & $32.7 \pm 2.9$ & $36.6 \pm 3.5$ \\
Sphere & $31.3 \pm 2.4$ & $34.7 \pm 2.5$ & $39.4 \pm 3.1$ \\
Pear & 26.6 & 27.8 & 33.2 \\
\hline
\end{tabular}



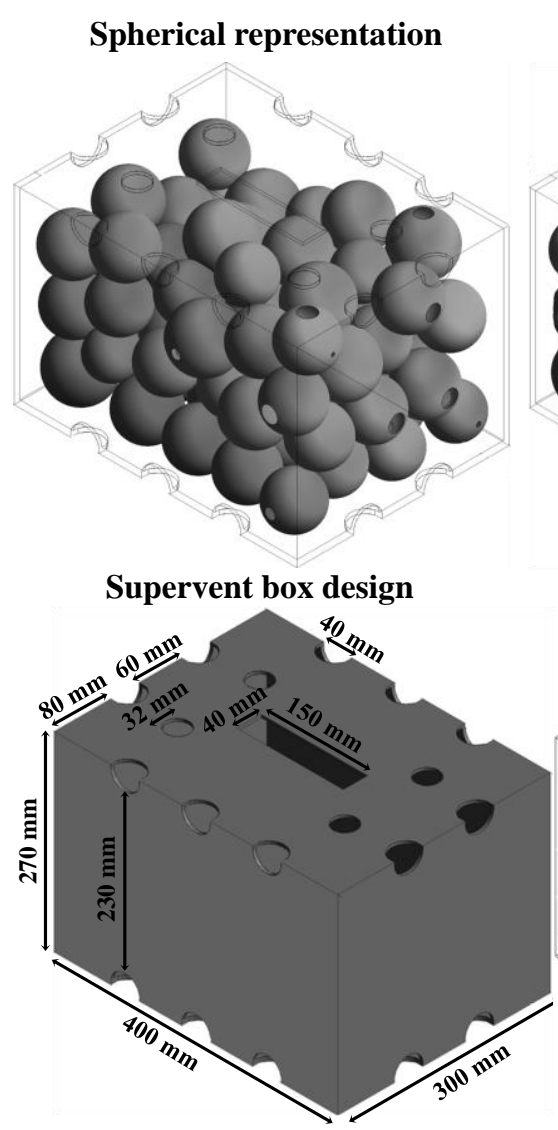

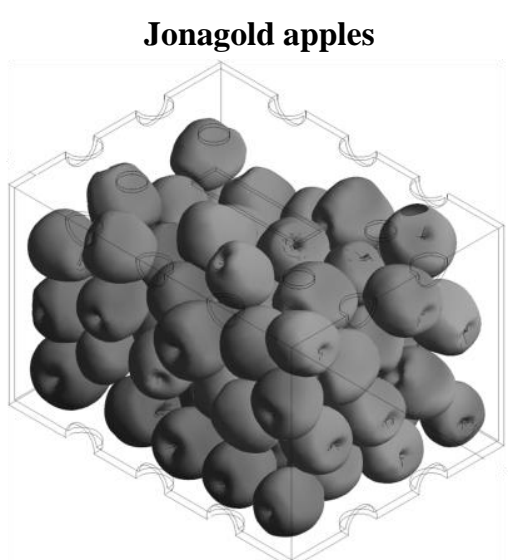

Conference pears

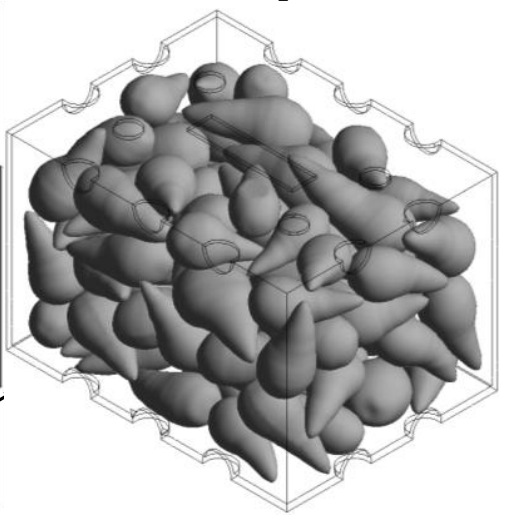

Mesh Jonagold apples

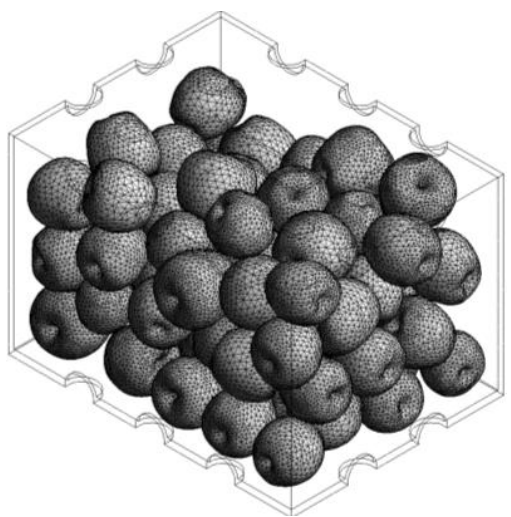

Mesh Conference pears

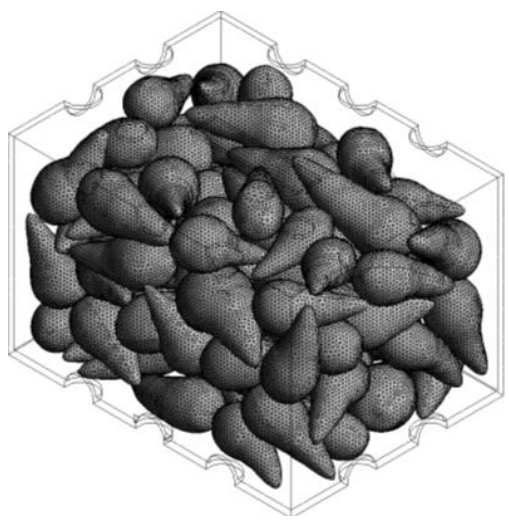

Figure 1: The geometrical illustration of the Supervent box design and an example of a stack filling generated using the Discrete Element Method of the conference pears, the Jonagold apples and the spherical representation of the apples. On the right, the mesh of an apple and pear filling is shown.

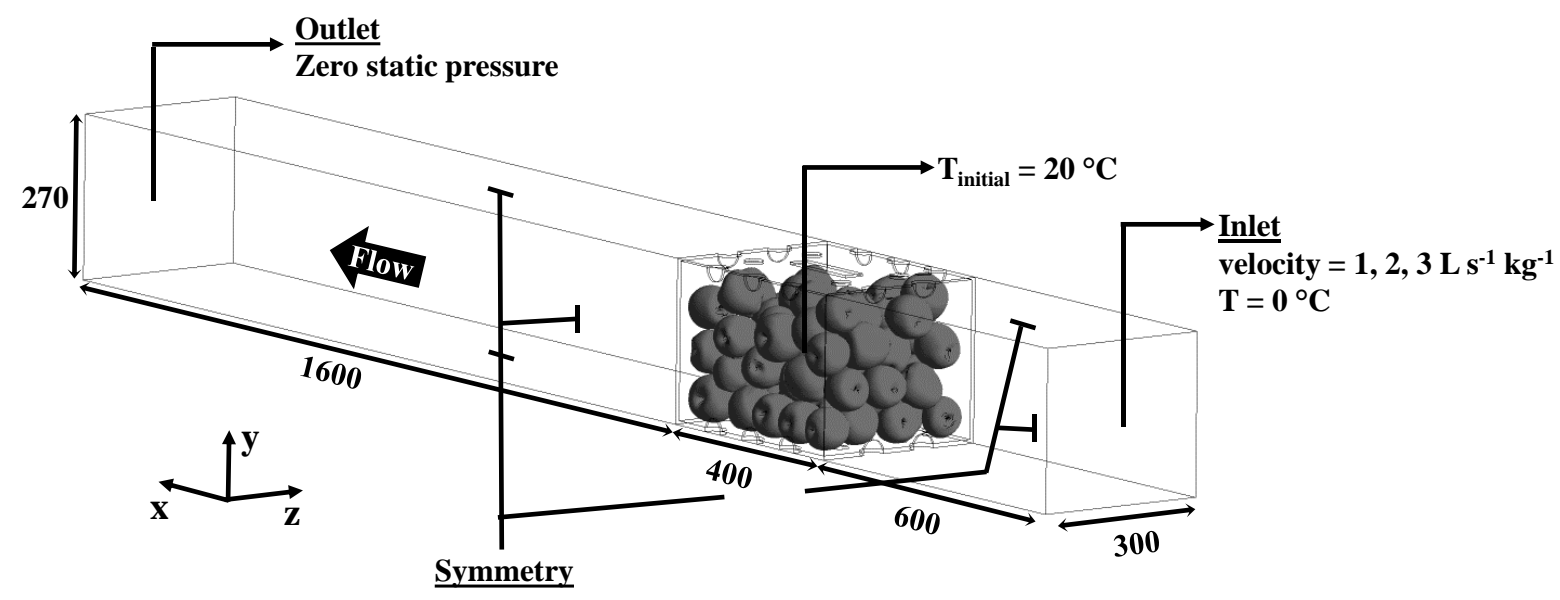

Figure 2: Schematic overview of the CFD model. The implemented boundary conditions were the same for the pear geometry, the three apple geometries and their corresponding spherical representations. The air enters the computational domain from the right hand side. The dimensions are expressed in $\mathbf{~ m m}$. 

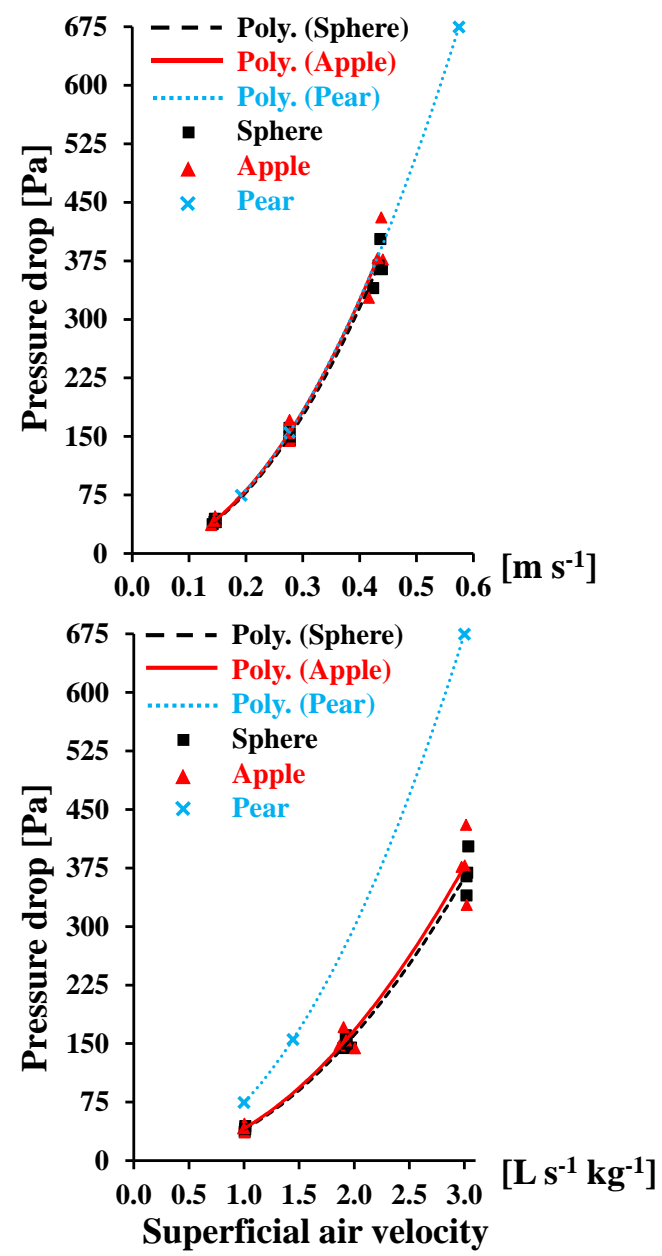

Figure 3: Pressure loss over the Supervent box filled with spheres (black), realistic apple (red) and pear shapes (blue) as a function of the superficial air velocity through the box, expressed in $\mathrm{m} \mathrm{s}^{-1}$ (top) and in $\mathrm{L} \mathrm{s}^{-1} \mathrm{~kg}^{-1}$ (bottom). The total mass of pear fruit was higher than that of apple fruit. As a consequence, the pressure loss characteristic for pears, expressed in $\mathbf{L ~ s}^{-1} \mathbf{k g}^{-1}$ (bottom), differed from the apple filling while it was the same when expressed in $\mathrm{m} \mathrm{s}^{-1}$ (top). The data points of the three fillings (squares and triangles for the spheres and apples, respectively) as well as the polynomial approximation (Poly.) of the average pressure loss are displayed. 


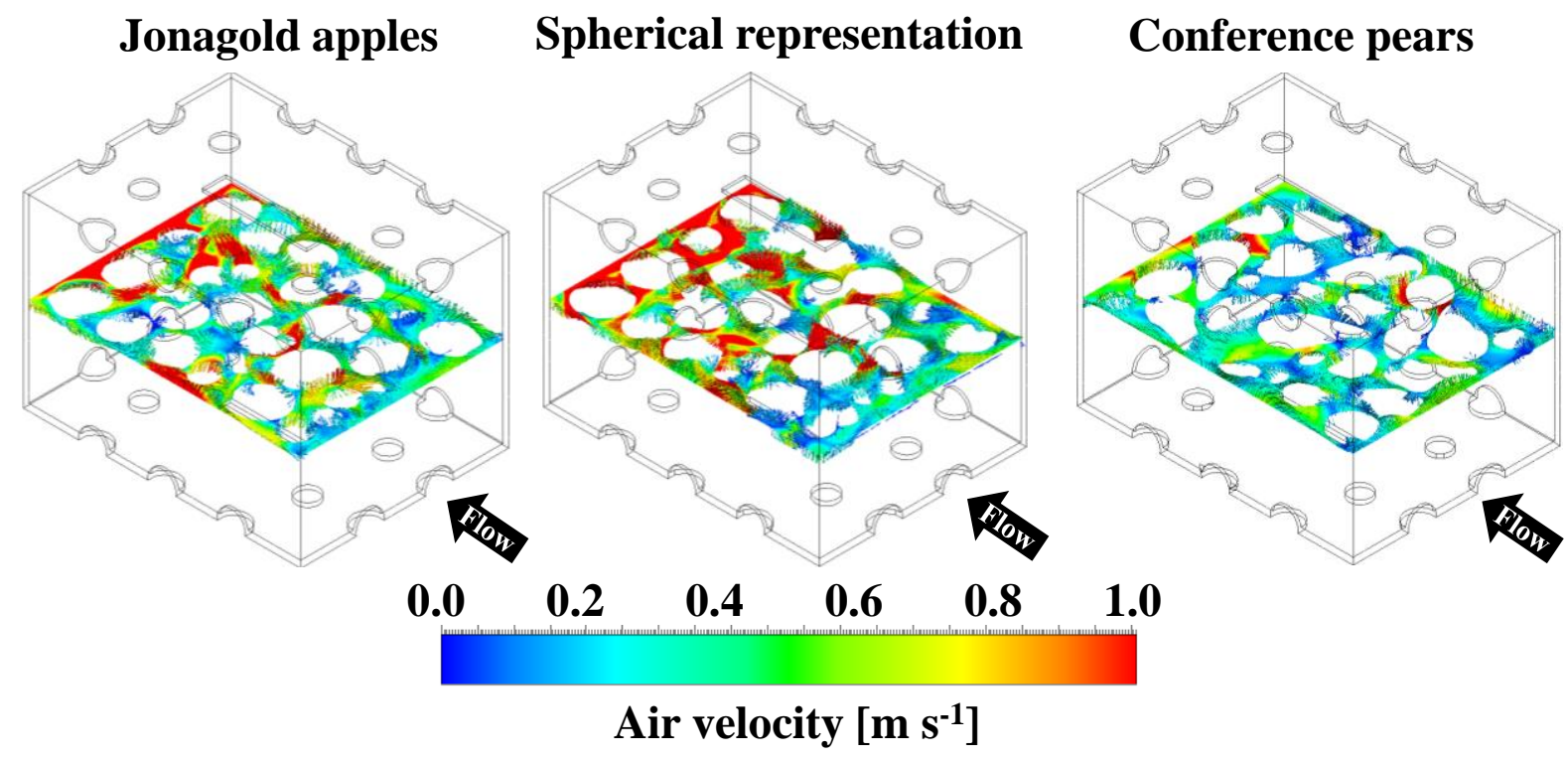

Figure 4: Velocity contour plots and velocity vectors for a medium flow rate in the middle of a box filled with apple shapes (left), equivalent spheres (middle) and pears (right). The air enters the computational domain from the right hand side.

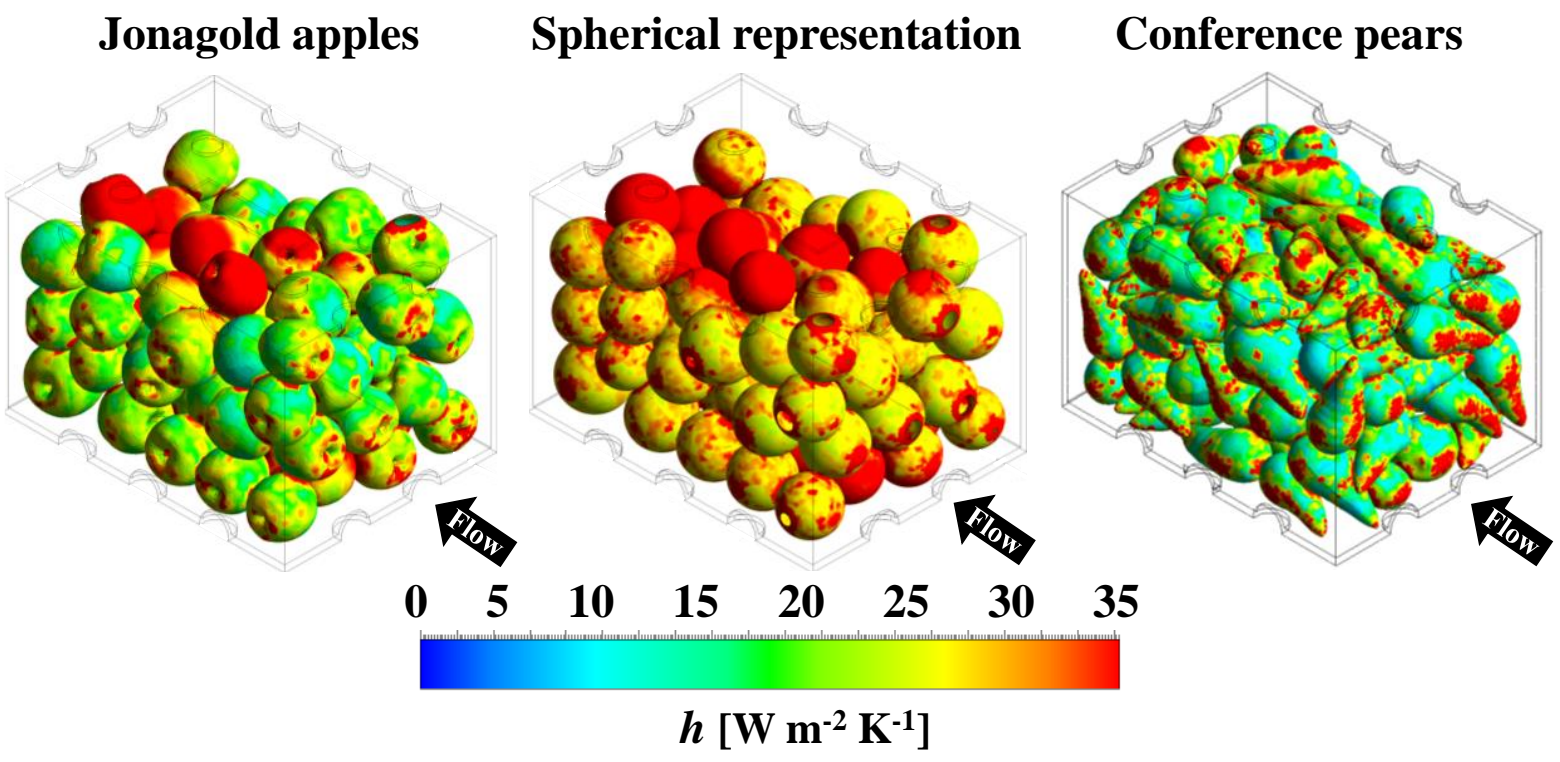

Figure 5: Contour plot of the convective heat transfer coefficients at the interface between the air and the fruit for a medium flow rate. The air enters the computational domain from the right hand side. 


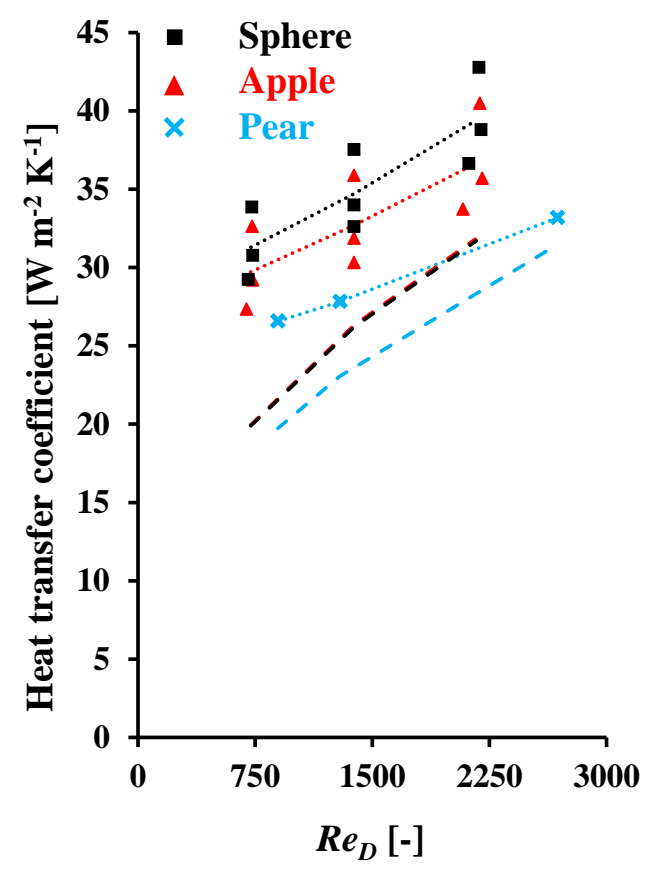

Figure 6: Surface averaged convective heat transfer coefficients $\left[\mathrm{W} \mathrm{m}^{-2} \mathrm{~K}^{-1}\right]$ as a function of particle Reynolds number for the pear, apple and spherical filling patterns. The dotted lines represent the average heat transfer coefficients from the three different fillings and are presented in Table 4. The dashed lines are the theoretical values, based on correlations for gas flow in a packed bed.
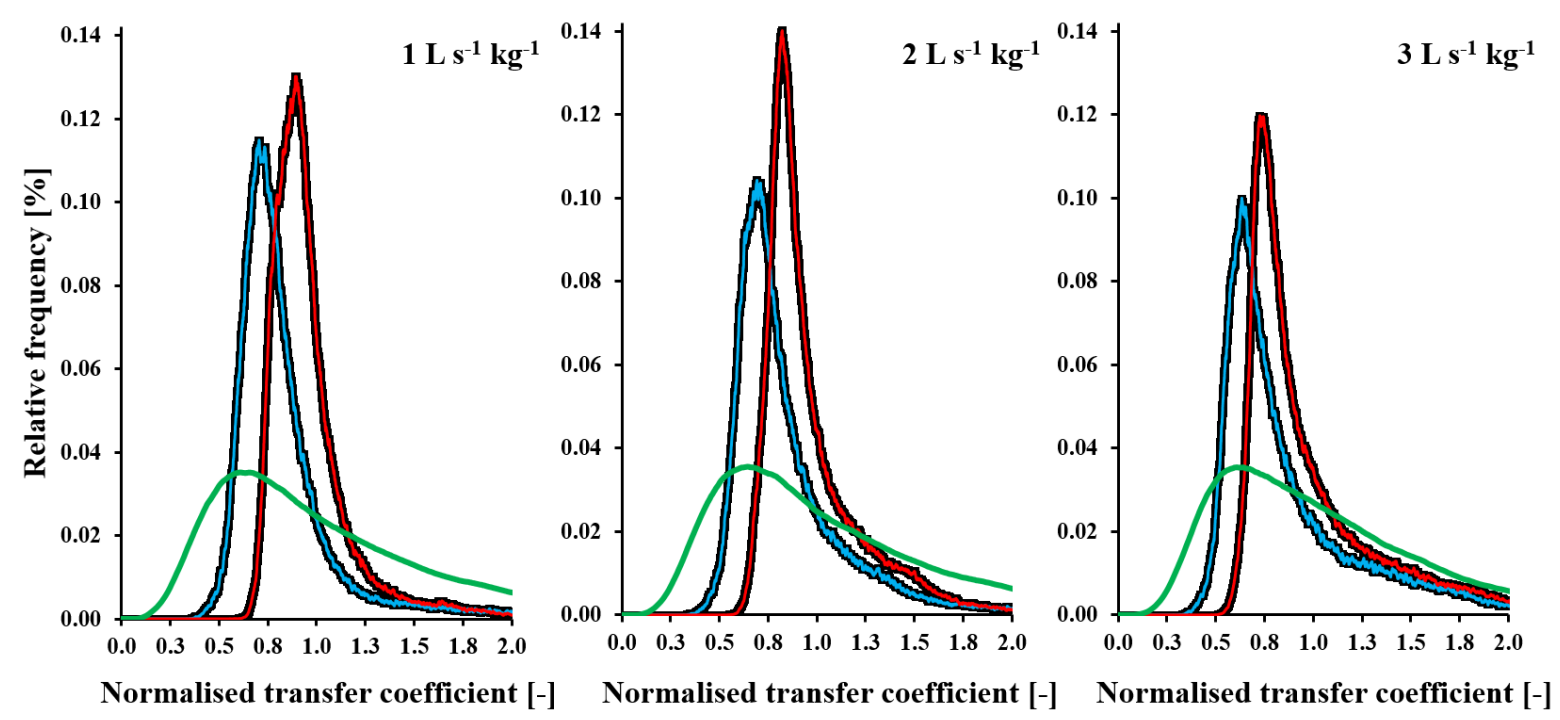

Figure 7: Average relative frequency distributions of the normalised convective heat transfer coefficients of all fruit in the Supervent box, with standard error ( 3 repetitions) for three different airflow rates (blue line for the apple shapes; red line for the spherical representation; green line for the pear shapes). The surface averaged convective heat transfer coefficient for each flow rate are presented in Table 4 and represent a normalised transfer coefficient of one. 


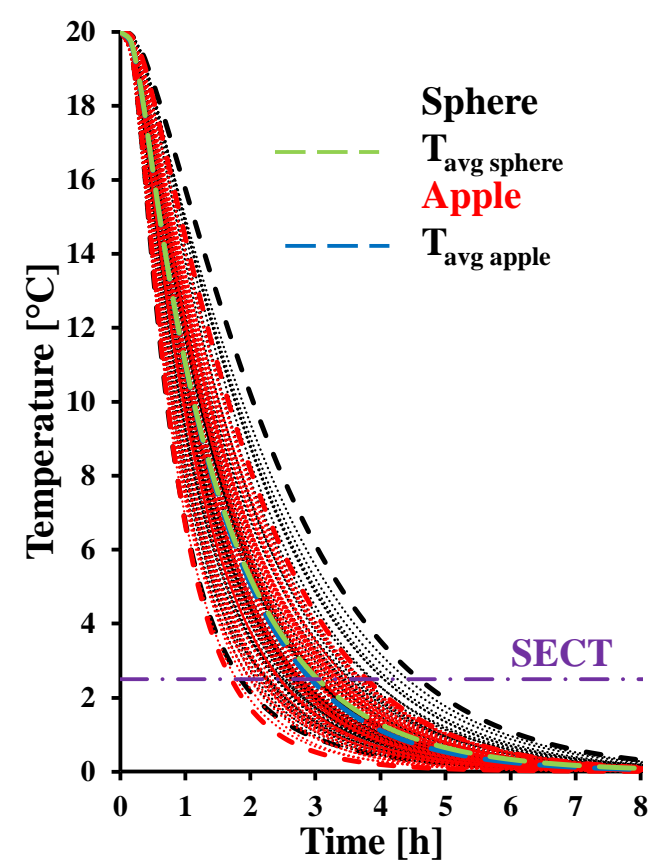

Figure 8: Time-temperature profiles calculated in the core of all fruit in the stack for the apple (red dots) and spherical representation (black dots) at medium airflow rate. The average temperature of all fruit in the box is depicted in blue and green dashes for the apples and spheres; the seven-eighths cooling time is depicted in purple. The minimum and maximum time-temperature profiles for both patterns are depicted in large dashes in the corresponding colours.

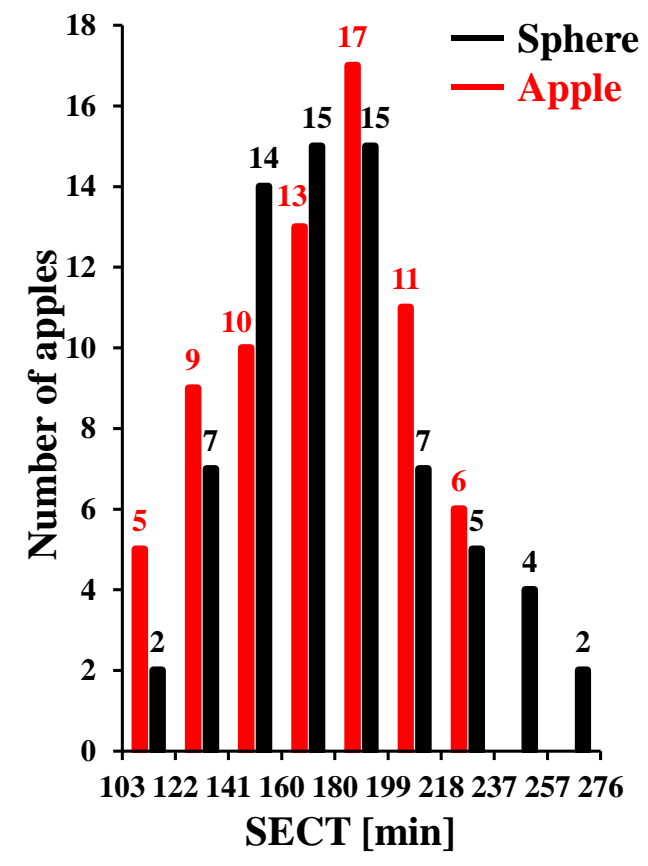

Figure 9: The distribution of the seven-eighths cooling time (SECT), expressed in minutes, calculated in the centre of each fruit in a stack of 71 fruit (apple indicated in red and its spherical representation indicated in black). 